\title{
O.S.P.
}

L'orientation scolaire et professionnelle

$48 / 4 \mid 2019$

Orientation et genre : approches psychosociales

\section{Les représentations professionnelles des étudiant·es en soins infirmiers : des représentations genrées}

Nursing students professional representations: gendered representations

Jean Maillet-Contoz, Gilles Combaz et Christine Morin-Messabel

\section{OpenEdition}

Journals

Édition électronique

URL : http://journals.openedition.org/osp/11551

DOI : 10.4000/osp. 11551

ISSN : 2104-3795

Éditeur

Institut national d'étude du travail et d'orientation professionnelle (INETOP)

Édition imprimée

Date de publication : 1 décembre 2019

Pagination : 581-609

ISSN : 0249-6739

Référence électronique

Jean Maillet-Contoz, Gilles Combaz et Christine Morin-Messabel, «Les représentations

professionnelles des étudiant·e.s en soins infirmiers : des représentations genrées », L'orientation

scolaire et professionnelle [En ligne], 48/4 | 2019, mis en ligne le 01 décembre 2021, consulté le 10 avril

2020. URL : http://journals.openedition.org/osp/11551 ; DOI : https://doi.org/10.4000/osp.11551

Ce document a été généré automatiquement le 10 avril 2020.

(c) Tous droits réservés 


\section{Les représentations professionnelles des étudiant·e's en soins infirmiers : des représentations genrées}

Nursing students professional representations: gendered representations

Jean Maillet-Contoz, Gilles Combaz et Christine Morin-Messabel

\section{Introduction}

1 La population des étudiante's en soins infirmiers comporte une répartition des sexes marquée par une très forte proportion de femmes : en 2016, elles représentaient $83,4 \%$ des étudiante-e's inscrite-s (DREES, 2018). La proportion d'hommes inscrits en formation a légèrement augmenté en quinze ans, passant de 12,3\% en 2004 (DREES, 2006) à 16,6\% en 2016. Cette distribution déséquilibrée entre les sexes trouve son origine dans la représentation sociale du métier d'infirmierère (Bosse \& Guégnard, 2007) d'une part, et dans la sexuation de l'orientation professionnelle (Vouillot, 2007), d'autre part. Si ces mécanismes d'orientation liés au genre sont bien identifiés (Mosconi, \& Stevanovic, 2014), nous souhaitons étudier en quoi les stéréotypes de sexe influencent la représentation que développent les étudiante-s en soins infirmiers de leur futur métier. Comment, une fois ces dernier'ère's entrée's en formation, les représentations professionnelles de leur métier se construisentelles et comment celles-ci s'imprègnentelles éventuellement d'assignations genrées?

\section{La formation actuelle des étudiant·e·s en soins infirmiers}

2 La dernière réforme des études en soins infirmiers date de 2009. La formation dure trois ans et se compose de 2100 heures d'enseignement au sein des instituts de formation en soins infirmiers (IFSI) et de 2100 heures de stage réalisées auprès 
d'infirmierère's sur différents terrains : soins généraux en milieu hospitalier, soins en psychiatrie, lieux de vie tels que les établissements d'hébergement pour personnes âgées dépendantes (EHPAD), soins à domicile, soins de longue durée ou encore soins de suite et de réadaptation. L'ensemble est organisé sous la forme d'une alternance intégrative.

3 La formation au sein des instituts est organisée en unités d'enseignement, empruntant aux sciences médicales, aux sciences humaines et sociales, au droit, à la philosophie et à l'éthique. Une partie des unités d'enseignement concerne spécifiquement le métier d'infirmierère dans ses dimensions techniques et organisationnelles, ainsi que dans le développement d'un raisonnement clinique spécifique.

4 L'encadrement en stage est assuré par des infirmierère's diplôméees d'État ; la formation à l'institut est assurée par des infirmierère's cadres de santé formateur.trice's, en collaboration avec d'autres professionnelle's et avec l'université de rattachement. Les évaluations réalisées au sein des unités d'enseignement et des stages permettent à l'étudiant·e d'acquérir les crédits européens (ECTS) nécessaires à l'obtention du diplôme d'État d'infirmierè̀re et de la licence en soins infirmiers.

5 Cette organisation de la formation a largement évolué tout au long du siècle dernier et s'inscrit dans une histoire au sein de laquelle la place des femmes et des hommes a connu beaucoup d'évolutions.

\section{La place des femmes et des hommes dans l'histoire des soins infirmiers}

$6 \mathrm{Si}$, jusqu'à la seconde moitié du XIX siècle, le soin est jugé comme une tâche dévalorisante, exercée de manière indifférenciée par des femmes ou des hommes, la profession infirmière connaît une féminisation, ainsi qu'une laïcisation au début du $\mathrm{XX}^{\mathrm{e}}$ siècle, en lien avec les valeurs de l'élite sociale d'alors. L'homme est renforcé dans son rôle de pourvoyeur économique au sein de la cellule familiale, en lien avec la première révolution industrielle (Christman, 1988), le développement de la classe ouvrière et l'exode rural.

7 Le soin constituant une activité professionnelle insuffisamment rémunératrice, les hommes s'en éloignent et les femmes s'y cantonnent.

8 La ségrégation des sexes dans l'univers du soin semble également pouvoir se rattacher aux prémices de la professionnalisation des infirmières. Ainsi, par exemple, Florence Nightingale, aristocrate anglaise, pionnière dans la création des soins infirmiers modernes, a contribué à conforter les femmes dans un rôle naturellement soignant, en extension de leurs rôles de mère et d'épouse. La profession d'infirmière offre aux femmes de l'époque la garantie d'un métier qualifié, hors de la sphère de la charité et de la domesticité (Cohen, 2000), loin de la rudesse du monde ouvrier, tout en demeurant extérieur au monde de la médecine, qui leur reste interdit (Roy, Holmes, \& Chouinard, 2011). Ce clivage entre médecine et soins infirmiers, comme celui entre femmes et hommes dans les soins infirmiers, semble également apparaître comme un fait récent dans l'histoire de la discipline (Schweitzera, 2009).

9 Les hommes se voient cantonnés au travail au sein des asiles, dans lesquels ils sont invités à mettre à profit leur force physique, afin de contenir les internés agités. Jusqu'en 1992, année de la disparition du diplôme d'infirmier spécialisé en psychiatrie, 
les hommes seront toujours plus présents dans les soins psychiatriques que dans les soins généraux.

Enfin, la lecture historique de la féminisation du soin infirmier est également renforcée par des travaux plus contemporains, comme ceux de Gilligan (1982), qui soutient l'idée d'une nature intrinsèquement féminine du care.

\section{Les représentations professionnelles}

11 Nous postulons que l'histoire des soins infirmiers influence encore largement les représentations sociales (Moscovici, 1961) du métier d'infirmierère, et également les représentations professionnelles. Ces dernières sont " des représentations sociales élaborées dans l'action et la communication professionnelle [...] et sont spécifiées par les contextes, les acteurs appartenant à des groupes et les objets pertinents et utiles pour l'exercice des activités professionnelles " (Blin, 1997, p. 80). Elles portent sur des objets spécifiques appartenant à une profession (Mias \& Piaser, 2016), et elles constituent un « lieu d'incorporation de la culture professionnelle propre aux groupes d'appartenance et de référence et intervenant dans la socialisation et dans la construction des identités professionnelles »(Blin, 1997, p. 54). Elles sont en interaction " avec les pratiques professionnelles car elles se construisent en fonction d'un référentiel commun au collectif de travail et servant de grille de lecture aux acteurs pour leur permettre de donner un sens, une signification à leurs activités et au contexte où ils agissent "(ibid.). Enfin, ces représentations sont partagées par une partie ou l'ensemble des membres de la profession. Elles présentent différents éléments descriptifs, prescriptifs, conditionnels ou évaluatifs (Mias \& Piaser, 2016).

12 À l'instar des représentations sociales, les représentations professionnelles ont des fonctions spécifiques au sein du groupe qui les partage :

- elles participent à la construction d'un savoir professionnel spécifique à l'exercice de la profession, elles garantissent les identités professionnelles,

- elles orientent les conduites et guident les pratiques professionnelles,

- dans l'exercice pratique, elles ont une double fonction d'orientation et de mobilisation des éléments cognitifs,

- elles permettent une justification, a posteriori, des pratiques professionnelles et des prises de position.

13 La première étape de l'analyse des représentations professionnelles repose sur le repérage de leurs contenus et repose sur l'analyse de trois dimensions (Blin, 1997) :

- une dimension fonctionnelle, incluant les objets activés dans l'activité professionnelle,

- une dimension contextuelle, à la fois organisationnelle (établissement d'exercice, appartenance à une équipe, statut des sujets...) et institutionnelle (idéologies et valeurs de l'organisation),

- une dimension identitaire, incluant les missions, les compétences, les qualités, les éléments motivationnels.

14 Les représentations professionnelles du métier d'infirmierère ont été abordées sous l'angle des liens entre le rôle propre, le rôle sur prescription médicale et les pratiques (Guimelli \& Jacobi, 1990). La projection dans le futur professionnel, en lien avec ces deux rôles (Guimelli, 1994) a également été traitée et reprise ensuite avec le prisme de la satisfaction professionnelle et de l'autonomie (Lheureux, 2010). Ces recherches ont 
également pu porter sur des objets spécifiques tels que l'hygiène hospitalière (Morlot et SalèsWuillemin, 2008). Ces études mobilisaient le test d'indépendance au contexte (TIC, Lo Monaco, Lheureux, \& Halimi- Falkowicz, 2008), la technique des schèmes cognitifs de base (SCB, Guimelli, 2003) ou encore celle d'association verbale à partir d'un mot inducteur (Abric, 2003).

\section{Les effets du genre sur les représentations professionnelles}

15 Les professions de la santé, fortement féminisées, ont favorisé l'étude de la place des femmes, des hommes et des effets du genre dans la sphère professionnelle, notamment chez les orthophonistes (Bretin \& Philippe, 2007), et les sages-femmes (Charrier, 2004 ; Jacques \& Purgues, 2012 ; Cottard, 2014). Ces études mettent en évidence les différences de motivation d'orientation entre femmes et hommes (Charrier, 2003), la persistance d'imaginaires genrés congruents à leur sexe chez des individus minoritaires sur le plan du sexe (Cottard, 2014) ou encore la " transformation » en compétences de qualités associées aux femmes (Jacques \& Purgues, 2012).

16 À travers les représentations professionnelles des futur·e's infirmierère's, nous souhaitons identifier les effets du genre mais également de l'appartenance à un groupe de sexe. Objet de nombreuses acceptions, le genre associe " les attributs psychologiques, les activités, les rôles et statuts sociaux culturellement assignés à chacune des catégories de sexe et constituent un système de croyances dont le principe d'une détermination est le pivot "(Hurtig, Kail, \& Rouche, 2002, p. 13). Cette détermination est illustrée par le sexe de l'individu et elle s'accompagne généralement d'une hiérarchisation (Mathieu, 2000). Le sexe est donc ici utilisé comme une variable tandis que le genre pourra constituer un facteur explicatif dans la variation des modalités de cette dernière.

17 Le genre influe sur les représentations sociales; ainsi, en lien avec une sensibilité accrue au contrôle social (Chappe, Brauer, \& Castano, 2004), les femmes développent généralement des représentations sociales plus homogènes que les hommes (LorenziCioldi, 1988).

18 Au travers des normes de genre, s'actualisent des stéréotypes de sexe, qui « reposent sur la mise en place d'antagonismes attribués quasi automatiquement au groupe des hommes et au groupe des femmes [...]. Ces traits attribués à l'un et à l'autre sexe reposent sur des dichotomies, des antagonismes, des oppositions. » (Gaborit, 2015, p. 19). Ainsi, Spence et Buckner (2000) identifient des traits considérés comme " communal » (ibid., p. 50) pour les femmes, c'est-à-dire relatifs à l'émotivité, à la focalisation sur la relation et la sensibilité envers autrui, et donc associant prioritairement la femme à la dimension du care. À l'inverse, les traits stéréotypiques masculins sont décrits comme " agentiques » (ibid.), désignant l'inclination à l'assertivité, la maîtrise et la focalisation sur une finalité ou un objectif précis.

19 Ces stéréotypes peuvent influer sur le choix de l'orientation professionnelle. L'analyse de l'orientation professionnelle différenciée entre filles et garçons montre ainsi combien les lycéen-ne's se projettent dans des métiers différents selon leur sexe (Mosconi \& Stevanovic, 2014). Les stéréotypes peuvent également, au sein d'une 
profession donnée, motiver une assignation différenciée des tâches entre les femmes et les hommes (Bereni et al., 2008).

\section{Hypothèses}

Nous souhaitons, dans cette recherche, étudier en quoi le genre peut influencer la construction des représentations professionnelles chez les étudiante's en soins infirmiers. Le travail ici présenté met à l'épreuve du réel l'hypothèse suivante : les étudiante's infirmierère's développent des représentations de leur futur métier, différentes selon leur sexe. Nous nous attendons donc à observer des différences dans les contenus et la structuration des représentations professionnelles chez les femmes et les hommes participant à l'étude. Sur le plan des hypothèses opérationnelles, elles peuvent donc se formuler ainsi :

- Au sein de la population des étudiante-s en soins infirmiers, les hommes développent des représentations professionnelles plus diversifiées que les femmes.

- Les femmes développent des représentations professionnelles davantage empreintes de stéréotypes féminins et parallèlement, les hommes développent des représentations professionnelles davantage empreintes de stéréotypes masculins.

21 Afin de tester ces deux hypothèses, deux études ont été menées, une première reposant sur la méthode de l'inductions hiérarchisée (Abric, 2003) et une seconde, plus courte et complémentaire, reposant sur une seconde méthodologie, le questionnaire de caractérisation (Guimelli, 1998).

\section{Première étude : l'évocation hiérarchisée}

\section{Méthode}

L'objet de cette étude est de modéliser les représentations professionnelles qu'ont les étudiante's en soins infirmiers de leur futur métier et d'étudier l'impact de leur appartenance de sexe sur ces représentations.

\section{Participant·es}

L'enquête a été réalisée auprès de 645 étudiante-s en soins infirmiers au sein de trois instituts de formation en soins infirmiers (IFSI). Les étudiante-s étaient inscrite-s en première année $(n=196)$, deuxième année $(n=196)$ et troisième année $(n=253)$. Sur l'ensemble des participante's, on retrouve $86,2 \%$ de femmes et $13,8 \%$ d'hommes, soit, pour ces derniers, une proportion légèrement inférieure à la moyenne nationale $(16,6 \%$ en 2016). L'âge moyen de l'ensemble des participantees est de 22,8 ans et l'âge médian de 21 ans. Les étudiante's étaient rencontrée's durant une période de cours et avaient la liberté de ne pas participer. Aucun refus n'a été constaté et l'ensemble des questionnaires recueillis a pu être exploité.

\section{Matériel}

24 Si l'approche initiale des représentations sociales est le fait de Moscovici (1961), la méthodologie ici retenue est celle de l'induction hiérarchisée (Abric, 2003), consistant, 
à partir d'un mot inducteur, à demander aux participantees de noter, de manière spontanée, les dix premiers mots qui leur viennent à l'esprit. Ces mots doivent ensuite être classés par ordre d'importance et éventuellement se voir attribuer une valence positive ou négative. Ce tout dernier point n'a pas été réalisé dans la présente étude. Le choix du mot inducteur, « infirmier » ou " infirmière », reposait sur sa pertinence. Ces deux inductions, selon leur genre grammatical, pouvaient l'une et l'autre générer des effets spécifiques (Stewart, 2004); dans le premier cas, l'utilisation du terme « infirmier " pouvait prendre deux significations, soit le professionnel de sexe masculin, soit le masculin neutre, avec le risque de modifier également l'évocation chez les participantes femmes. Dans le second cas, les participants hommes de l'échantillon pouvaient ne pas s'identifier au mot inducteur et cela serait venu stimuler chez eux des représentations sociales plutôt que des représentations professionnelles. Il a donc été fait le choix de les utiliser l'un et l'autre, de manière aléatoire.

\section{Procédure}

Pour chaque année de formation, l'échantillon a donc été séparé en deux groupes, pour lequel le mot inducteur a été «infirmière » $(n=291)$ ou «infirmier» $(n=354)$. Un support de recueil adapté a été fourni aux participant·es. Le recueil s'est déroulé de la manière suivante :

- La recherche a été présentée comme un travail de thèse portant sur le thème des étudiante-s en soins infirmiers.

- L'énoncé de la première consigne était « À partir d'un mot inducteur, vous noterez les mots ou expressions qui vous viennent à l'esprit (dix maximum)».

- Le mot inducteur était projeté sur un écran et les étudiante-s disposaient de trois minutes pour répondre.

- La seconde consigne, demandant de hiérarchiser les mots par ordre d'importance, était ensuite énoncée. Cette seconde phase durait également trois minutes.

- Il leur était ensuite demandé de noter leur âge ainsi que leur sexe.

Cette procédure a permis le recueil d'un corpus de mots, chacun assorti d'une pondération. Ce corpus a tout s'abord été soumis à une analyse lexicométrique, puis ses éléments principaux ont permis d'élaborer un schéma de la représentation professionnelle, et enfin, ces derniers ont été soumis à une analyse factorielle de correspondances, à l'aide du logiciel SPaD (Coheris Analytics SPAD)

\section{Résultats}

L'ensemble des participante's a ainsi produit au total, 5790 mots, dont 704 mots différents. Chez les étudiantes, on dénombre un total de 4966 mots pour 616 mots différents. Ces derniers sont au nombre de 278 chez les étudiants, sur un total de 824 mots exprimés (voir tableau 1 ).

Tableau 1 : Analyse lexicométrique du corpus Table 1: Corpus lexicometric analysis

\begin{tabular}{|l|l|l|l|}
\hline & Total & \multicolumn{2}{|l|}{ Selon le sexe } \\
\hline & Echantillon complet & Femmes & Hommes \\
\hline
\end{tabular}




\begin{tabular}{|l|l|l|l|}
\hline Total des occurrences & 5790 & 4966 & 824 \\
\hline Nombre de types* & 704 & 616 & 278 \\
\hline Nombre de hapax* & 386 & 331 & 169 \\
\hline Indice de diversité & .12 & .13 & .34 \\
\hline Indice de variabilité & .55 & .54 & .61 \\
\hline Nombre de non-réponses & 660 & 594 & 66 \\
\hline Indice non-réponse/total des occurrences & .11 & .12 & .08 \\
\hline
\end{tabular}

*le nombre de types et de hapax est calculé au sein de chaque sous-partie de l'échantillon (les femmes, les hommes) Un hapax identique dans le sous-groupe des femmes et celui des hommes n'est plus un hapax dans l'échantillon complet. Ainsi, pour les types et les hapax, les données de l'échantillon complet ne sont pas un cumul des colonnes situées plus à droite.

\section{Analyse lexico-métrique}

Les différences de construction des représentations professionnelles peuvent s'envisager dans leur dimension sémantique mais également dans leur « topographie lexicale » (Kalampalikis \& Moscovici, 2005, p. 19). Le corpus recueilli a donc tout d'abord fait l'objet d'une analyse lexico-métrique. Les trois indicateurs retenus ici sont (ibid.) :

- le volume du corpus et le nombre d'occurrences de chaque mot,

- le nombre de types, c'est-à-dire le nombre de mots différents,

- le nombre de hapax : le nombre de mots uniques, donc cités une seule fois et par une seule participante.

Ces trois indicateurs permettent de calculer deux indices de structuration du corpus (Flament \& Rouquette, 2003) :

- L'indice de diversité : basé sur le rapport types/occurrences, il oscille entre 0 et 1 . S'il est égal à 1 , cela signifie qu'aucun mot n'est répété, témoignant d'une grande richesse lexicale dans la population étudiée ou d'une grande hétérogénéité de cette dernière.

- L'indice de variabilité : basé sur le rapport hapax/types, il oscille également entre 0 et 1 . Plus sa valeur est proche de 1 , plus certaines sources du corpus se distinguent des autres par le lexique employé.

Un troisième indice est ici ajouté afin de considérer les non-réponses, pour les participante-s ayant exprimé moins de dix mots ; une participante qui, par exemple, n'aurait exprimé que sept mots est supposée générer trois non-réponses.

Dans le tableau 1, le calcul de l'indice de diversité indique une hétérogénéité nettement plus grande de la représentation chez les hommes (.34) que chez les femmes (.13); de même, cette population renvoie un indice de variabilité indiquant davantage de distinction entre les hommes (.61) qu'entre les femmes (.54). Enfin, le pourcentage de non-réponse est $50 \%$ plus élevé chez ces dernières. Le sexe des participant·e's semble ici constituer une variable tout à fait significative. 


\section{Schématisation des représentations professionnelles}

Abric (2003) propose ensuite de classer les termes jugés significatifs en quatre groupes, selon la fréquence et l'importance de la pondération :

- Les éléments fréquents et importants appartiennent au noyau central,

- Les éléments fréquents mais peu importants constituent la première périphérie, c'est-à-dire les éléments périphériques les plus importants,

- Les éléments peu fréquents et importants sont dits " éléments contrastés " et peuvent constituer un sous-groupe porteur d'une représentation minoritaire,

- Enfin, les éléments peu fréquents et peu importants constituent la deuxième périphérie.

Les tableaux 2 et 3 présentent les différents éléments constitutifs des représentations professionnelles selon le sexe des participante.s. On constate tout d'abord que pour les deux sexes, le contenu sémantique du noyau central est identique. Il contient majoritairement des éléments axés sur l'altérité incarnée par le "patient»: «relationnel», « accompagnement ", « écoute», « empathie». Le mot «soin» est probablement issu de la représentation sociale du métier d'infirmierère (Stewart, 2004). De même, les éléments de la première périphérie sont identiques chez les femmes et les hommes; trois de ces éléments sont relatifs au contexte d'exercice professionnel : «blouse », « hôpital », « équipe ».

Des différences entre les sexes apparaissent au sein des éléments contrastés et de la deuxième périphérie. Dans les éléments contrastés, les termes apparaissant dans les deux groupes sont relatifs à des qualités ("respect», "bienveillance») et des compétences («collaboration»), à l'orientation vers autrui («soignant», « humain ») et à un des concepts fondamentaux des soins infirmiers, la «santé ». Les termes apparaissant seulement dans le groupe des hommes sont également relatifs à l'altérité («aidant », «social») et incluent également " paramédical ». Le groupe des femmes valorise quant à lui les qualités de "patience » et de «rigueur», le "soutien» et la " vocation ». Ce dernier mot est cité par $8,8 \%$ des femmes contre $2,2 \%$ des hommes.

Enfin, la deuxième périphérie agrège de manière consensuelle, chez les deux sexes, les termes généraux de "métier » et de "travail » et trois objets renvoyant à des actes courants chez les infirmierère's : "pansement », «médicament » et "piqûre ». Les femmes ajoutent des éléments de compétence ("organisation») et de contexte ( "maladie », " service», « médecin»), tandis que les hommes y valorisent les termes « urgence » et « diplôme ».

Tableau 2 : Structure de la représentation professionnelle du métier chez les étudiantes en soins infirmiers

Table 2: Structure of Female Nursing Students professional representation

\begin{tabular}{|l|l|l|}
\hline \multirow{2}{*}{$|l| l \mid$} \\
\cline { 2 - 3 } & IMPORTANCE & FAIBLE $(<6,1)$ \\
\cline { 2 - 3 } & NOYTE $(>6,1)$ & PREMIÈRE PÉRIPHÉRIE \\
\hline
\end{tabular}




\begin{tabular}{|c|c|c|}
\hline $\begin{array}{l}\text { FRÉQUENCE } \\
\text { FORTE } \\
(>18,1 \%)\end{array}$ & $\begin{array}{l}\text { soin }(80,9 \% ; 8,4) \\
\text { relationnel }(36,0 \% ; 7,8) \\
\text { patient }(24,7 \% ; 7,0) \\
\text { accompagnement }(22,5 \% ; 6,4) \\
\text { responsabilité }(20,2 \% ; 8,0) \\
\text { empathie }(20,2 \% ; 6,5) \\
\text { écoute }(19,1 \% ; 7,8)\end{array}$ & $\begin{array}{l}\text { hôpital }(39,3 \% ; 3,9) \\
\text { équipe }(30,3 \% ; 5,7) \\
\text { blouse }(27,0 \% ; 3,9) \\
\text { technique }(19,1 \% ; 5,2)\end{array}$ \\
\hline & ÉLÉMENTS CONTRASTÉS & DEUXIÈME PÉRIPHÉRIE \\
\hline $\begin{array}{l}\text { FRÉQUENCE } \\
\text { FAIBLE } \\
(<18,1 \%)\end{array}$ & $\begin{array}{l}\text { santé }(15,7 \% ; 8,0) \\
\text { soignant }(11,2 \% ; 8,9) \\
\text { collaboration }(7,9 \% ; 6,3) \\
\text { bienveillance }(6,7 \% ; 8,3) \\
\text { paramédical }(6,7 \% ; 6,0) \\
\text { aidant }(6,7 \% ; 7,5) \\
\text { respect }(5,6 \% ; 6,6) \\
\text { humain }(5,6 \% ; 9,6) \\
\text { social }(5,6 \% ; 6,2)\end{array}$ & $\begin{array}{l}\text { travail }(13,5 \% ; 4,8) \\
\text { piqûre }(12,4 \% ; 3,6) \\
\text { pansement }(11,2 \% ; 4,3) \\
\text { médicament }(10,1 \% ; 4,8) \\
\text { urgence }(9,0 \% ; 4,0) \\
\text { diplôme }(6,7 \% ; 4,0) \\
\text { métier }(5,6 \% ; 3,6)\end{array}$ \\
\hline
\end{tabular}

Note : les nombres écrits entre parenthèses sont le pourcentage d'évocation et le rang moyen d'importance. Ainsi, par exemple, soin $(82,4 \% ; 8,4)$ signifie que le mot soin a été évoqué par $82,4 \%$ des participantes et associé par elles à un rang moyen d'importance de 8,4 .

Tableau 3 : Structure de la représentation professionnelle du métier chez les étudiants en soins infirmiers

Table 3: Structure of Male Nursing Students professional representation

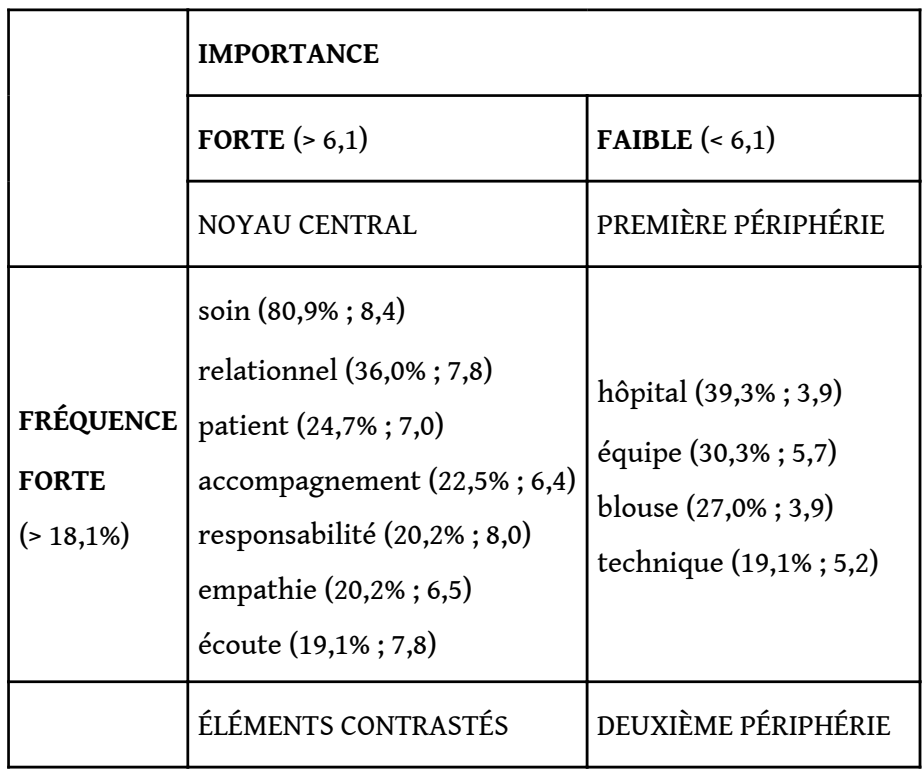




\begin{tabular}{|l|l|l|}
\hline & santé $(15,7 \% ; 8,0)$ & \\
& soignant $(11,2 \% ; 8,9)$ & travail $(13,5 \% ; 4,8)$ \\
FRÉQUENCE & collaboration $(7,9 \% ; 6,3)$ & piqûre $(12,4 \% ; 3,6)$ \\
FAIBLE & bienveillance $(6,7 \% ; 8,3)$ & pansement $(11,2 \% ; 4,3)$ \\
$(<18,1 \%)$ & paramédical $(6,7 \% ; 6,0)$ & médicament $(10,1 \% ; 4,8)$ \\
& aidant $(6,7 \% ; 7,5)$ & urgence $(9,0 \% ; 4,0)$ \\
& respect $(5,6 \% ; 6,6)$ & diplôme $(6,7 \% ; 4,0)$ \\
& humain $(5,6 \% ; 9,6)$ & métier $(5,6 \% ; 3,6)$ \\
\hline
\end{tabular}

Note : les nombres écrits entre parenthèses sont le pourcentage d'évocation et le rang moyen d'importance. Ainsi, par exemple, soin $(80,9 \% ; 8,4)$ signifie que le mot soin a été évoqué par $80,9 \%$ des participants et associé par eux à un rang moyen d'importance de 8,4.

\section{Analyse factorielle des trente éléments principaux}

Une analyse factorielle des correspondances multiples (ACM) a ensuite été réalisée sur les trente mots les plus cités, et ayant une pondération moyenne supérieure à la moyenne de l'ensemble des pondérations, soit 5,5. Les données ont fait l'objet d'un recodage, chaque mot étant considéré comme une variable active à deux modalités : expression du mot ou non-expression du mot. Les données concernant le sexe des participant·es, leur âge et leur année d'études ont été traitées comme des variables supplémentaires (Escofier et Pagès, 1988), n'influant pas sur la construction du nuage de points.

L'ACM permet d'identifier 22 axes nécessaires pour expliquer $80 \%$ de la variance. $\mathrm{Au}$ vu de leur qualité de représentation graphique, les axes 1 et 3 , expliquant à eux deux $10,4 \%$ de la variance, ont été retenus pour l'analyse.

La représentation graphique (voir figure 1) ne conserve que les modalités « expression d'un mot» afin d'en faciliter l'interprétation. Du fait de leur important effectif, la majorité des modalités «non-expression d'un mot » se situait au centre du graphique, avec donc peu de significativité.

Tout d'abord, au niveau des variables, l'axe 3, en ordonnée, semble opposer un pôle "vocation » et un pôle " passion ». Le pôle " vocation », situé dans la partie positive de l'axe, comprend des termes tels que " prise en charge », "soigner ", " santé », ainsi que des éléments évoquant les individus (« humain »), malades (« malade», «patient») ou professionnels ("soignant», «équipe»). Il est donc fortement axé sur l'altérité, incluant soignants et soignés, et sur une définition large du métier d'infirmier à travers l'acte de soigner et la promotion de la santé.

Le second pôle, situé dans la partie inférieure de l'axe 3, inclut des qualités personnelles : « bienveillance », " patience », « respect », « douceur », « attention », des compétences professionnelles : "empathie », « rigueur », " écoute », " confiance » et enfin des termes renvoyant également à l'altérité tels que " contact », "relationnel », « accueil », « soutien » mais dans une dimension plus opérationnelle et incluant à la fois soignant et soigné.

Sur un plan horizontal, l'axe 1 comporte sur sa gauche de nombreux éléments renvoyant à l'interaction interhumaine, renvoyant au soigné (" patient»), au collectif 
soignant (" équipe ») ou à "l'humain » et à la relation qui les lie, notamment à travers quatre éléments comportant tous le préfixe co- (cum, avec, en latin) : « collaboration », « confiance », « contact » et « communication ». La partie droite de l'axe regroupe les qualités et les compétences citées précédemment ainsi que "responsabilité », « altruisme » et « courage ».

Il semble également possible de regrouper les mots dans trois espaces principaux, basés sur les différentes dimensions des représentations professionnelles (Blin, 1997) : une zone contextuelle, basée sur les rôles généraux du personnel soignant et les finalités du soin, une seconde zone contextuelle mais plutôt axée sur la dimension interhumaine du soin, avec les notions d'équipe et de patient ; enfin, une troisième zone regroupe des éléments se rattachant à la dimension identitaire de la profession, à travers l'énoncé de qualités et de compétences. La position des différents participant·es, selon leur sexe, au sein de ces trois ensembles peut orienter notre analyse.

Figure 1 : Éléments du noyau central (analyse des correspondances multiples, plans 1 et 3) Elements of the central core (muliple correspondence analysis, plans 1 and 3)

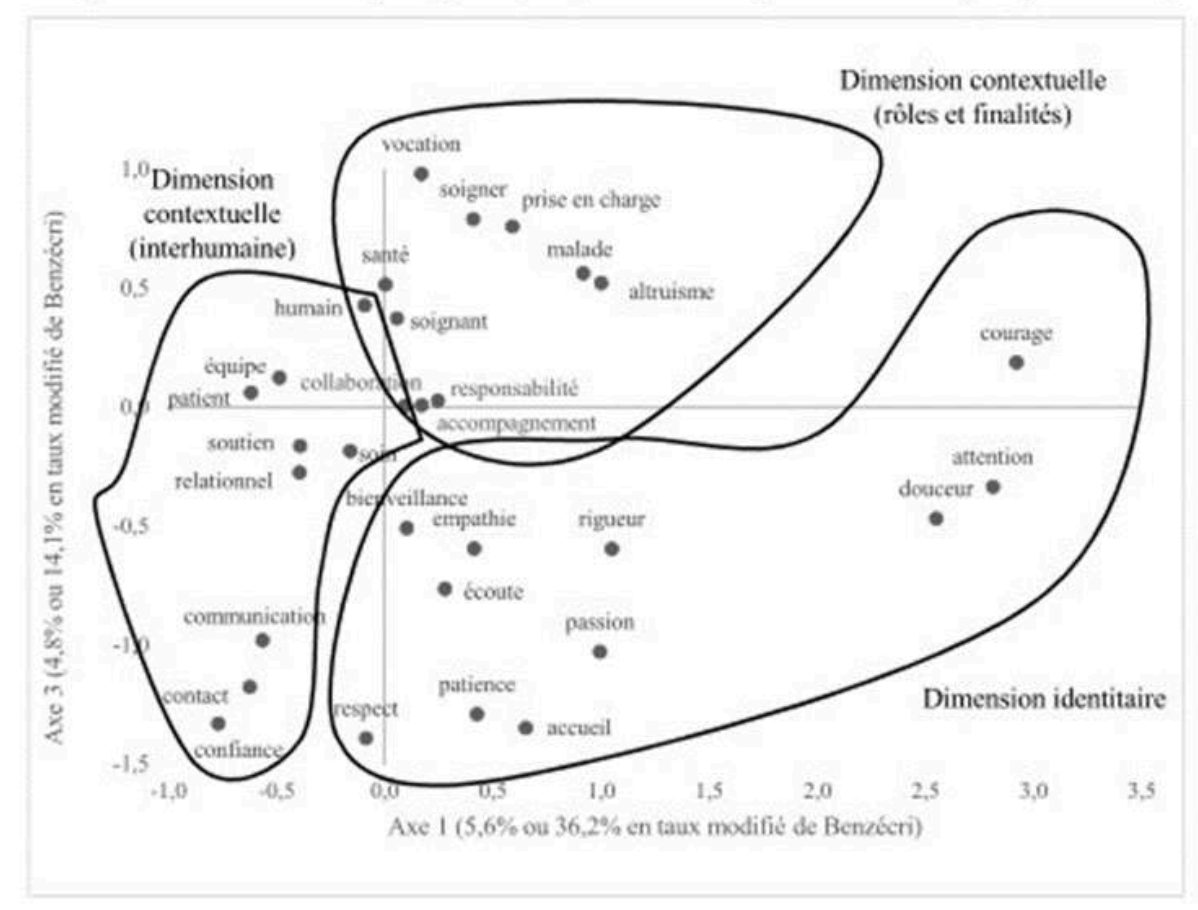

\section{Analyse du nuage des participant $\cdot e \cdot s$}

Afin de faciliter à la fois la lecture et l'interprétation, le nuage des participant-e-s est illustré sous forme de figures, elles-mêmes complétées par des tableaux présentant le nombre d'individus présent dans chacune des quatre parties du graphe. Les deux graphes (voir figures 2 et 3 ) illustrent respectivement la répartition de l'ensemble des participante-s sur les deux plans, puis uniquement le groupe des hommes. La comparaison des deux figures permet de visualiser spatialement la différence de distribution des individus selon leur sexe. La comparaison des tableaux 5 et 6 permet de mettre en évidence les différences de répartition des femmes et des hommes. 
Figure 2 : Répartition des participant $\cdot e \cdot s$ (analyse des correspondances multiples, plans 1 et 3 ) Participant distribution (muliple correspondence analysis, plans 1 and 3)

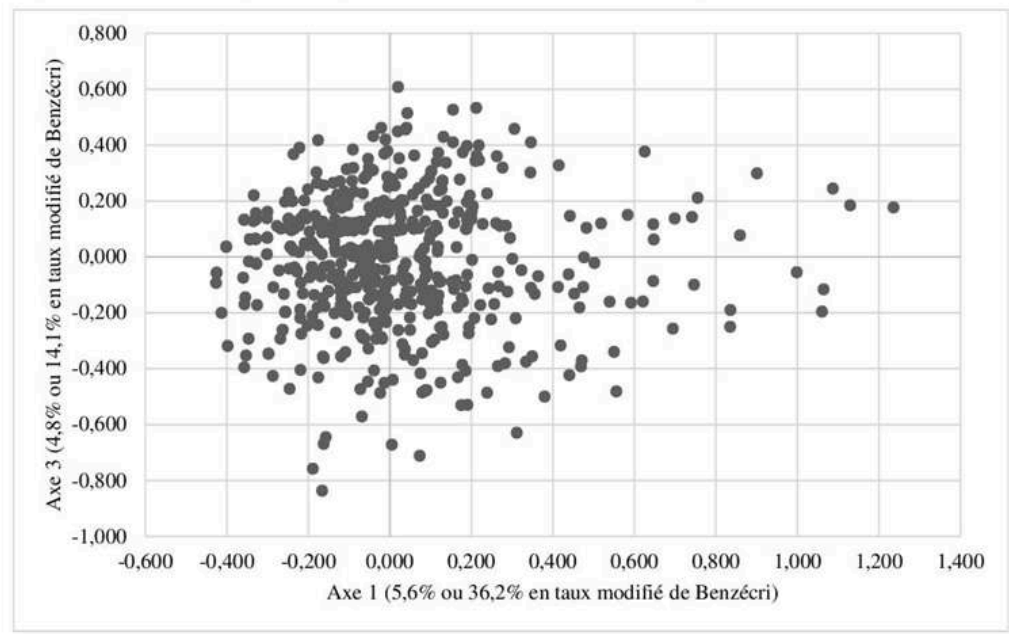

Figure 3 : Répartition des participants de sexe masculin (analyse des correspondances multiples, plans 1 et 3)

Male participant distribution (muliple correspondence analysis, plans 1 and 3)

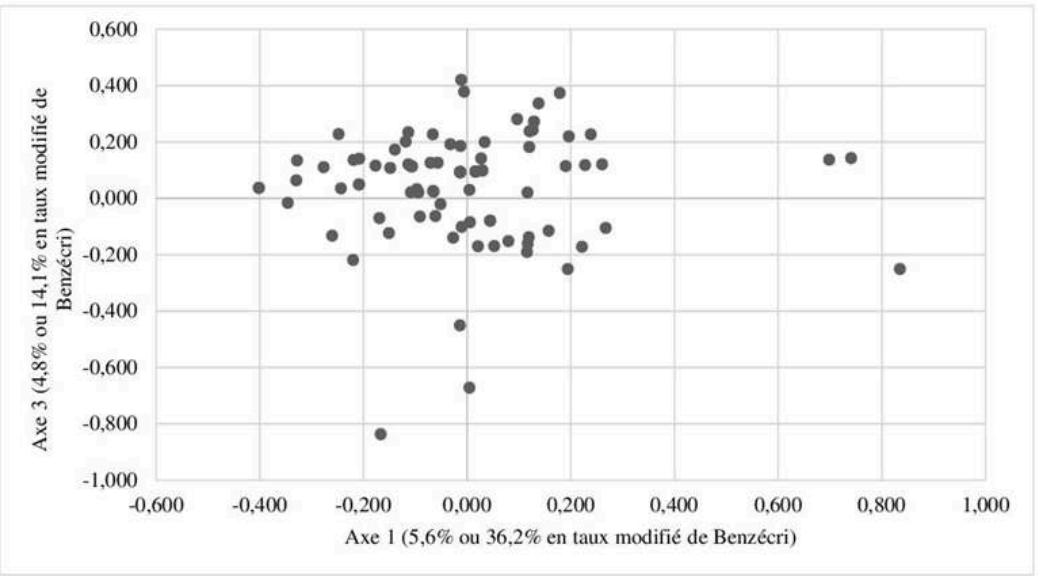

44 Au niveau de l'ensemble des participant·es, le nuage de points (voir figure 2) présente une forme symétrique entre le haut le bas de l'axe 3. La proportion de participant·e's dans les quatre zones du graphe est indiquée dans le tableau 4. Sur l'axe 1, se répartissent $45 \%$ des participant·e's, très concentrée'es à gauche, et $55 \%$, sensiblement plus étalée's, à droite. Cette répartition des individus prend tout son intérêt en faisant apparaître le sexe de ces derniers.

Tableau 4 : Répartition des participant·e.s selon la polarité des axes Table 4 : Participant distribution according to axis polarity

\begin{tabular}{|l|l|l|l|l|}
\hline \multicolumn{2}{|c|}{} & \multicolumn{2}{|l|}{ Axe 1 } & \\
\cline { 3 - 5 } \multicolumn{2}{|c|}{} & négatif & positif & total \\
\hline Axe 3 & positif & $34.40 \%$ & $20.80 \%$ & $55,20 \%$ \\
\hline
\end{tabular}




\begin{tabular}{|l|l|l|l|l|}
\cline { 2 - 4 } & négatif & $25.40 \%$ & $19.40 \%$ & $44,80 \%$ \\
\hline & total & $59,80 \%$ & $40,20 \%$ & $100 \%$ \\
\hline
\end{tabular}

En effet, si l'on considère la variable supplémentaire du sexe des participant·es, on observe que les femmes se répartissent à $60,4 \%$ sur la gauche de l'axe 1 (voir tableau 5). En revanche, les hommes sont plus nombreux, 69,6\%, dans la partie supérieure de l'axe 3 , indiquant soit une tendance à s'écarter des qualités et des compétences situées dans la partie inférieure du graphe, soit à se rapprocher de la dimension plutôt contextuelle du métier d'infirmier (voir tableau 6).

Tableau 5 : Répartition des femmes selon la polarité des axes Table 5 : Female participant distribution according to axis polarity

\begin{tabular}{|l|l|l|l|l|}
\hline \multicolumn{2}{|c|}{} & \multicolumn{2}{l|}{ Axe 1 } & \\
\cline { 2 - 5 } \multicolumn{2}{|c|}{} & négatif & positif & total \\
\hline \multirow{2}{*}{ Axe 3 } & positif & $33.10 \%$ & $19.80 \%$ & $52,90 \%$ \\
\cline { 2 - 5 } & négatif & $27.30 \%$ & $19.80 \%$ & $47,10 \%$ \\
\hline & total & $60,40 \%$ & $39,60 \%$ & $100 \%$ \\
\hline
\end{tabular}

La répartition des individus montre donc qu'une majorité d'hommes semble développer une représentation plutôt abstraite, orientée sur la mission globale de l'infirmier’ère, qui est de "soigner ", d'assurer une "prise en charge ", de promouvoir la "santé ", avec une identification claire du "soignant» et du «malade». Les femmes, quant à elles, ont une répartition plus équilibrée au sein des différents espaces représentationnels.

Tableau 6 : Répartition des hommes selon la polarité des axes Table 6: Male participant distribution according to axis polarity

\begin{tabular}{|l|l|l|l|l|}
\hline \multicolumn{2}{|c|}{} & \multicolumn{2}{l|}{ Axe 1 } & \\
\cline { 3 - 5 } \multicolumn{2}{|c|}{} & négatif & positif & total \\
\hline \multirow{2}{*}{ Axe 3 3} & positif & $42.70 \%$ & $26.90 \%$ & $69,60 \%$ \\
\cline { 2 - 5 } & négatif & $13.50 \%$ & $16.90 \%$ & $30,40 \%$ \\
\hline & total & $56,20 \%$ & $43,80 \%$ & $100 \%$ \\
\hline
\end{tabular}




\section{Seconde étude : le questionnaire de caractérisation}

\section{Méthode}

47 Les résultats obtenus précédemment ont été soumis à une seconde méthode, le questionnaire de caractérisation (Guimelli, 1998), notamment pour confirmer le statut central des éléments identifiés comme tels.

\section{Participants}

Le questionnaire a été adressé aux étudiant·es d'un institut de formation. L'enquête a permis le recueil de 102 questionnaires auprès de participante's de première année ( $\mathrm{n}=$ $33)$, deuxième année $(n=36)$ et troisième année $(n=33)$, dont $84,8 \%$ de femmes et $15,7 \%$ d'hommes.

\section{Matériel}

49 À partir des éléments recueillis lors de l'enquête par évocation hiérarchisée, un questionnaire de caractérisation a été élaboré en mêlant des éléments identifiés comme centraux, contrastés et appartenant à la première et à la deuxième périphérie. Les mots ont été regroupés en cinq groupes de six mots. Pour chaque groupe de mots, la consigne était la suivante : "Cocher les deux mots qui caractérisent le mieux votre futur métier d'infirmier(e). Cocher également les deux mots qui caractérisent le moins votre futur métier d'infirmier(e)».

\section{Procédure}

Les questionnaires ont été diffusés par voie électronique, en vue d'une autoadministration.

51 On obtient ainsi pour chaque mot un score pour les deux items suivants : "très important» et «peu important». Lorsque le mot n'est identifié ni dans l'un ni dans l'autre item, il est alors qualifié de « moyennement important».

À partir des scores obtenus, il est possible d'établir, pour chaque mot, trois types de profil :

- Les mots désignés majoritairement comme très importants correspondent à des éléments centraux.

- Les mots désignés majoritairement comme moyennement ou peu importants sont des éléments périphériques.

- Les éléments ayant donné lieu à des jugements opposés sur le plan de l'importance, entre peu et très importants, sont considérés comme contrastés.

\section{Résultats}

Le questionnaire de caractérisation confirme, pour les deux sexes, le statut central de l'ensemble des éléments identifiés comme tels dans l'étude précédente (tableaux 2 et 3 ) : soin, relationnel, écoute, accompagnement, responsabilité et empathie. Seul le mot « patient " est renvoyé à un statut périphérique chez les femmes et les hommes. Il est possible que ce mot ait souffert de sa double acception, en tant qu'adjectif renvoyant à 
une qualité humaine, et en tant que nom, correspondant à un usager du système de soin. C'est dans cette dernière acception qu'il était proposé, tout en ayant pu être compris selon la première. Le mot " écoute " apparaît également ici comme périphérique, mais uniquement chez les hommes. Par ailleurs, ce questionnaire invite à considérer comme centraux, dans les deux sous-échantillons, les mots « collaboration ", « humain », « bienveillance » et « respect ».

Cette seconde étude permet donc, à une exception près, de confirmer le statut des éléments centraux de la représentation. De plus, elle raccroche au noyau central quatre autres éléments, dont trois renvoient à des qualités personnelles et le dernier à une compétence professionnelle.

\section{Discussion générale} ailleurs, ces résultats sont conformes à ce qui a été mis en évidence au sein de la population générale par Lorenzi-Cioldi (1988) : le groupe des femmes, socialement dominé, développe, d'une manière générale, des représentations sociales plus homogènes que celui des hommes, socialement dominant. Les membres des groupes dominés auraient une plus grande tendance au conformisme du fait d'une sensibilité accrue au contrôle social (Chappe, Brauer \& Castano, 2004), tandis que ceux du groupe dominant pourraient s'autoriser davantage de singularité dans leurs prises de positions. Il s'agit là d'un premier point notable, les représentations professionnelles des étudiants en soins infirmiers de sexe masculin sont plus diversifiées, dans leur contenu, que celles des étudiantes de sexe féminin et par ailleurs, les étudiants de sexe masculin expriment davantage de singularité que leurs consœurs.

La seconde hypothèse opérationnelle était que les femmes développent des représentations professionnelles davantage empreintes de stéréotypes féminins, et les hommes, de stéréotypes masculins. L'aCM, réalisée sur les mots issus du noyau central, permet d'identifier trois pôles principaux, celui de la dimension contextuelle 
interhumaine, de la dimension contextuelle axée sur le rôle soignant et ses finalités et celui de la dimension identitaire, rassemblant les qualités et les compétences attendues d'un " bon " infirmier ou d'une "bonne " infirmière. Ces éléments permettent d'envisager la modélisation de certains idéaux-types du·de la futur·e soignant·e :

- l'infirmierère valorisant la dimension collaborative et relationnelle de son métier,

- l'infirmier-ère axée sur son statut de soignantee, à travers une vocation de soin et de promotion de la santé,

- l'infirmierère se reconnaissant dans les qualités personnelles et les compétences professionnelles investies dans l'acte de soin.

60 On note des différences entre les participant·es femmes et hommes ; la répartition des individus sur la projection du nuage montre qu'une majorité d'hommes semble développer une représentation davantage abstraite, orientée sur la mission globale de l'infirmierère, qui est de "soigner ", d'assurer une "prise en charge ", de promouvoir la « santé », avec une identification claire du « soignant » et du « malade ». Les femmes, quant à elles, investissent davantage les différents espaces représentationnels, au sein desquels leur répartition est plus équilibrée. Comme pour les hommes, mais dans une proportion bien moindre, elles sont, malgré tout, nombreuses à se rapprocher d'un pôle marqué par les buts et les finalités du soin.

61 Ces observations sont conformes à des travaux décrivant les éléments stéréotypiques d'une forme de masculinité, dans le fait de considérer un ensemble plutôt que les détails (Spence et Buckner, 2000), de nommer précisément les choses et en particulier les places de chacun (Brugère, 2006). Les hommes paraissent s'éloigner d'une représentation qui serait très empreinte de qualités stéréotypées à la fois comme féminines (Williams et Best, 1977) et soignantes, la douceur, la patience, l'attention ou la bienveillance.

Du point de vue de la structure sémantique il est intéressant de considérer les similitudes et les différences entre les deux groupes de sexe. On observe ainsi, de manière consensuelle, chez les étudiante's infirmierère's une présence forte des dimensions associées à la relation au patient : relationnel, patient, écoute, accompagnement, humain. Les quatre premiers mots apparaissent, en outre, dans le noyau central. Cet aspect relationnel, s'il s'inscrit dans le cadre du rôle propre infirmier, serait également une forme de délégation de la part du corps médical (Molinier, 2014). Le soin relationnel et éducatif apparaît également comme très important chez les professionnelle's diplômée's (Lheureux, 2010). Au niveau des différences sur le plan relationnel, le groupe des femmes valorise le terme " soutien » tandis que celui des hommes met en avant le « social ", nettement plus neutre.

Les qualités identifiées consensuellement - empathie, bienveillance, respect - se réfèrent à un registre stéréotypique féminin, tandis que la compétence de responsabilité renvoie, quant à elle, à un registre davantage masculin. Ici, la qualité est féminine, tandis que la compétence, supposant un apprentissage reconnu, est plutôt masculine. Les travaux cités précédemment montrent également comment les professionnelles de santé font de ces qualités, des compétences revendiquées (Jacques et Purgues, 2012), afin de mieux tenir éloignées leurs identités de femme et de professionnelle (Cottard, 2014). D'un point de vue différentiel, les hommes valorisent la qualité " aidant " contre celle de " patience ", plus féminine, chez les femmes. Ces dernières mettent également en avant d'autres compétences : la rigueur et l'organisation. Enfin, sans être totalement une qualité mais plutôt une forme de 
prérequis fantasmatique, la vocation apparaît au sein du groupe féminin, et avec elle le stéréotype de la religieuse infirmière dévouée à Dieu et à autrui.

Enfin, au sein des deux groupes, la première et la deuxième périphérie agrègent, entre autres, des éléments relatifs au rôle technique sur prescription - technique, piqûre, médicament, pansement - à la dimension interprofessionnelle - équipe, collaboration et au contexte de travail - hôpital, blouse, métier, travail - constituant la partie " négociable » de la représentation (Moliner et Guimelli, 2015) et permettant l'adaptation de l'individu au contexte (ibid.). La première périphérie est identique dans les deux groupes, en revanche, la seconde met en évidence des contrastes entre les sexes ; les femmes y valorisent trois termes, "maladie ", "médecin » et "service», fortement ancrés dans une réalité hospitalière, tandis que l'on retrouve chez les hommes le terme "diplôme ", renvoyant plutôt à leur condition estudiantine, et celui d'« urgence", constituant un secteur d'activité prisé par eux.

Ainsi, sur la base de l'analyse de l'aCM et de l'analyse structurale, il semble possible de valider la seconde hypothèse opérationnelle, relative à l'activation de stéréotypes sexués congruents au sexe des participants, au sein de leurs représentations professionnelles.

\section{Conclusion}

Cette recherche est basée sur une exploration des représentations professionnelles des étudiante's en soins infirmiers, en lien avec l'appartenance de sexe des participante-s.s. Elle s'appuie sur une méthode d'induction hiérarchisée, produisant un corpus dont se dégage, d'une part, une schématisation des représentations professionnelles qu'ont, de leur futur métier, les étudiants en soins infirmiers. La centralité des éléments du noyau de cette représentation a fait l'objet d'une confirmation à l'aide d'un questionnaire de caractérisation

D'autre part, l'ensemble de ce corpus a fait l'objet d'une analyse lexicométrique, et les éléments identifiés comme appartenant au noyau central ont été modélisés au sein d'une analyse statistique multidimensionnelle. Cette double approche permet d'identifier certaines différences dans les prises de position entre les femmes et les hommes engagée'es dans la formation en soins infirmiers.

Les différences constatées portent à la fois sur l'organisation et les contenus des représentations. Ces dernières apparaissent plus larges et diversifiées chez les hommes, phénomène connu pour les représentations sociales appartenant à un groupe traditionnellement placé en situation de domination. Concernant les contenus des représentations professionnelles, il semble que, au sein du groupe des femmes, ils soient plus empreints de qualités et de compétences attribuées de manière stéréotypique à ce groupe. À l'inverse, il semble que le groupe des hommes développe beaucoup moins cette vision traditionnelle d'une soignante pétriee de qualités maternelles et de compétences essentialisées. Par ailleurs, le groupe des hommes développe majoritairement des contenus axés sur les finalités et les objectifs des soins, renvoyant là encore à une dimension stéréotypique.

69 L'hypothèse d'une construction différenciée des représentations professionnelles, révélée par l'appartenance de sexe, apparaît donc, à ce stade, pertinente. 
70 Le travail ici exposé comporte plusieurs limites. Tout d'abord, il réduit les participant·e's à leur sexe. L'analyse est donc basée sur la binarité femme-homme sans considérer d'autre particularité. La prise en compte des éléments tels que leur biographie, leur socialisation ou encore les motivations ayant présidé à leur orientation, sera tout à fait opportune dans un second temps afin de mettre également en évidence les éventuelles différences intra-sexes.

71 Ensuite, elle ne permet pas aux participants de justifier leurs prises de positions et d'exposer plus précisément leur pensée. Ainsi, cette étude pourra s'enrichir d'une série d'entretiens semi-directifs avec des étudiant·es. L'approche des représentations professionnelles par le biais de ces entretiens et l'analyse du discours des étudiant·e's qui suivra permettraient également d'adopter une méthode d'exploration plus en lien avec la théorie initiale de Moscovici (1961) concernant les représentations sociales. Ce seront ainsi les contenus des représentations, l'attitude des étudiant·e·s concernant ceux-ci et également leur hiérarchisation qui pourraient être révélés.

72 Enfin, sur un plan statistique, le choix a été fait, pour cette étude, de faire participer des promotions entières, sans chercher à équilibrer le nombre de femmes et d'hommes. Cela aurait introduit un biais que les participant·es auraient immédiatement détecté, avec le risque de modifier la spontanéité de leurs réponses. Les entretiens devraient donc reposer sur un échantillonnage stratifié (Blanchet \& Gotman, 2010), qui permettra de rencontrer un nombre équivalent de participant·ess des deux sexes.

73 Précisons enfin qu'un tel travail peut sembler adopter une vision très différentialiste des groupes de sexe, voire chercher à renforcer les différences perçues entre ces groupes, notamment dans la sphère professionnelle. C'est, cependant, une démarche bien différente qui anime cette recherche, cherchant à identifier et à objectiver l'influence des assignations genrées dans la production de ces différences, et donc à mettre en lumière des points aveugles dans la socialisation professionnelle et le futur exercice d'un métier très marqué par sa répartition des sexes.

L'éventualité d'une construction différenciée des représentations professionnelles, chez les étudiant·es, intéresse l'ingénierie de la formation elle-même et notamment les résistances rencontrées par les chargé'e's de formation et d'orientation, que ce soit en formation initiale ou plus tard, dans le choix d'une spécialisation. Enfin cette réflexion peut contribuer à élaborer et à mettre en œuvre des leviers pertinents destinés à œuvrer pour l'égalité entre les sexes au sein de la formation.

\section{BIBLIOGRAPHIE}

Abric, J.-C. (2003). La recherche du noyau central et de la zone muette des représentations sociales. In J.-C. Abric (Ed.), Méthodes d'études des représentations sociales, (pp. 59-80). Toulouse, France : Érès. DOI: 10.3917/eres.abric.2003.01.0003

Bereni, L., Chauvin, S., Jaunait, A., \& Revillard, A. (2008). Introduction aux gender studies. Bruxelles, Belgique : De Boeck. 
Blanchet, A., \& Gotman, A. (2015). L'entretien. Paris, France : armand Colin.

Blin, J.-F. (1997). Représentations, pratiques et identités professionnelles. Paris, France : L’Harmattan.

Bretin, H., \& Philippe, C. (2007). Les hommes orthophonistes. In L. Tain (Ed.), Le métier

d'orthophoniste : langage, genre et profession (pp. 137145). Rennes, France : Editions ENSP.

Bosse, N., \& Guégnard, C. (2007). Les représentations des métiers par les jeunes : entre résistances et avancées. Travail, genre et sociétés, 18(2), 27-46. DOI: 10.3917/tgs.018.0027

Brugère, F. (2006). La sollicitude. La nouvelle donne affective des perspectives féministes. Esprit, 2006(1), 123-140. DOI: 10.3917/espri.0601.0123

Chappe, B., Brauer, M., \& Castano, E. (2004). Advantaged groups are more variable than disadvantaged groups: the case of preference and habits. Cahiers de psychologie cognitive, 22(4-5), 407-425. Repéré à https://www.researchgate.net/ publication/278817770

Charrier, P. (2004). Comment envisage-t-on d'être sage-femme quand on est un homme? L'intégration professionnelle des étudiants hommes sage-femmes. Travail, genre et sociétés, 2(12), 105-124. DOI: $10.3917 /$ tgs.012.0105

Chiland, C. (2003). Le transsexualisme, Paris, France : Presses Universitaires de France.

Christman, L. (1988). Men in nursing. Annual Review of Nursign Research, 6, 193-205. DOI: 10.1891/0739-6686.6.1.193

Cohen, Y. (2000). Profession infirmière. Une histoire des soins dans les hôpitaux du Québec. Montréal, Québec : Les presses de l'Université de Montréal.

Cottard, L. (2014). Homme et sagefemme : un choix, une pratique, une construction identitaire. Nouvelle revue de psychosociologie 17(1), 97-108. DOI: 10.3917/nrp.017.0097

DREES Direction de la Recherche, des Études, de l'Évaluation et des Statistiques (2006). Les étudiants en formation paramédicale en 2004. Études et résultats, 540. https://drees. solidaritessante.gouv.fr/etudeset-statistiques/publications (page consultée le 18 février 2019).

DREES Direction de la Recherche, des Études, de l'Évaluation et des Statistiques (2018). La formation aux professions de santé en 2016. Série "Statistiques ", 205. https://drees.solidaritessante.gouv.fr/etudeset-statistiques/publications (page consultée le 18 février 2019).

Escofier, B., \& Pagès, J. (1988). Analyses factorielles simples et multiples. Objectifs, méthodes et interprétation. Paris, France : Dunod.

Flament, C., \& Rouquette, M.-L. (2003). Anatomie des idées ordinaires. Paris, France : Armand Colin. Gaborit, P. (2015). Les stéréotypes de genre. Identités, rôles sociaux et politiques publiques. Paris, France : L'Harmattan.

Gilligan, C. (1982). Une si grande différence. Paris, France : Flammarion.

Guimelli, C., \& Jacobi, D. (1990). Pratiques nouvelles et transformation des représentations sociales. Revue Internationale de Psychologie Sociale, 3(3), 307-334.

Guimelli, C. (1994). La fonction d'infirmière. Pratiques et représentations sociales. In J.-C. Abric (Ed.), Pratiques sociales et représentations (pp. 83-108). Paris, France : Presses Universitaires de France.

Guimelli, C. (1998). Chasse et nature en Languedoc :étude de la dynamique d'une représentation sociale. Paris, France : L'Harmattan. 
Guimelli, C. (2003). Le modèle des Schèmes Cognitifs de Base (SCB) : méthodes et applications. In J.-C. Abric (Ed.), Méthodes d'études des représentations sociales, (pp. 119-143). Toulouse, France : Érès. DOI: 10.3917/eres.abric.2003.01.0119

Hurtig, M.-C., Kail, M., \& Rouch, H. (2002). Sexe et genre. De la hiérarchie entre les sexes. Paris, France : CNRS.

Jacques, B., \& Purgues, S. (2012). L'entrée des hommes dans le métier de sagefemme : faire sa place dans un monde professionnel « ultraféminisé ». Revue française des affaires sociales, 2, 52-71. Repéré à : https://www.cairn. inforevue-francaise-des-affairessociales-2012-2-page-52.htm.

Kalampalikis, N., \& Moscovici, S. (2005). Une approche pragmatique de l'analyse ALCESTE. Les Cahiers Internationaux de Psychologie Sociale, 66, 15-24. DOI: 10.3917/ cips.066.0015

Lheureux, F. (2010). Représentations professionnelles, satisfaction au travail et choix de carrière des personnels infirmiers : le rôle des valeurs d'autonomie. Psychologie du Travail et des Organisations, 16(4), 312-325. DOI: 10.1016/S14202530(16)30139-X

Lo Monaco, G., Lheureux, F., \& HalimiFalkowicz, S. (2008). Test d'indépendance au contexte (TIC) et structure des représentations sociales. Swiss Journal of Psychology, 67(2), 119-123. DOI: 10.1024/1421-0185.67.2.119

Lorenzi-Cioldi, F., (1988). Individus dominants et groupes dominés : images masculines et féminines. Grenoble, France : Presses Universitaires de Grenoble.

Mathieu, N.-C., (2000). Sexe et genre. In H. Hirata. (Ed.), Dictionnaire critique du féminisme (pp. 205-213). Paris, France : Presses universitaires de France.

Mias, C., \& Piaser, A. (2016). Les représentations professionnelles. In G. Lo Monaco, S. Delouvée, \& P. Rateau (Eds.), Les représentations sociales. Théories, méthodes et applications (pp. 319-322). Louvainla-Neuve, Belgique : De Boeck.

Moliner, P., \& Guimelli, C. (2015). Les représentations sociales. Grenoble, France : Presses Universitaires de Grenoble.

Molinier, P. (2014). Les métiers ont-ils un sexe ? In M. Fournier (Ed.). Masculinféminin. (pp. 196-204). Paris, France : Éditions Sciences Humaines. Repéré à https:// www.cairn.infomasculinfeminin---page-196.htm

Morlot, R., \& Sales-Wuillemin, E. (2008). Effet des pratiques et des connaissances sur la représentation sociale d'un objet : application à l'hygiène hospitalière. Revue internationale de psychologie sociale, 4 (tome 21), 89-114. Repéré à http://www.cairn.info/revueinternationale-depsychologie-sociale-2008-4-page-89.htm

Mosconi, N., \& Stevanovic, B. (2014). Genre et avenir. Les représentations des métiers chez les adolescentes et les adolescents. Paris, France : L'Harmattan.

Moscovici, S. (1961). La psychanalyse, son image et son public. Paris, France : PUF.

Roy, B., Holmes, D., \& Chouinard, V. (2011). Contribution à une éthique de la sollicitude. Masculinités et genre dans la profession infirmière. Recherche en soins infirmiers, 107, 38-48. DOI: $10.3917 /$ rsi.107.0038

Schweitzera, S. (2009). Du vent dans le ciel de plomb, L'accès des femmes aux professions supérieures, $\mathrm{XIX}^{\mathrm{e}}-\mathrm{XX}{ }^{\mathrm{e}}$ siècles. Sociologie du travail, 51, 183-198. DOI: 10.4000/sdt.16383

Spence, J.-T., \& Buckner, C.-E. (2000). Instrumental and expressive traits, traits stereotypes and sexist attitudes: what do they signify? Psychology of Women Quarterly, 24, 44-62. DOI: 10.1111/j. 1471-6402.2000.tb01021.x 
Stewart, I. (2004). La représentation sociale de la profession d'infirmier(ière) : effets de contexte liés à un changement de genre. Psychologie et société, 4(1), 171-209.

Vouillot, F. (2007). L'orientation aux prises avec le genre. Travail, genre et sociétés, 18, 87-108. DOI: $10.3917 /$ tgs.018.0087

Williams, J.-E., \& Best, D.-L. (1977). Sex stereotypes and trait favorability on the adjective check list, Educational and psychological measurement, 37, 101-110. DOI: 10.1177/001316447703700111

\section{RÉSUMÉS}

Les études en soins infirmiers attirent très majoritairement des femmes. L'objectif de la présente recherche est de modéliser les représentations professionnelles que développent les étudiant·e·s en soins infirmiers de leur futur métier, et d'étudier l'impact du genre sur la construction de ces représentations. Une méthode d'évocation par induction hiérarchisée, complétée par un questionnaire de caractérisation, permet de révéler des différences entre les participant·es femmes et hommes. Ces différences portent sur la structure des représentations, plus large concernant les hommes. De même, les contenus, comportent des contenus sexo-stéréotypés : les qualités chez les femmes et les finalités des soins infirmiers pour les hommes.

Nursing studies attract mostly women. This research's aim is to model professional representations that nursing students develop during their studies. It also aims to learn about the impact of gender on representation construction. An evocation method by hierarchical induction, completed by a characterization questionnaire, reveals differences between female and male participants. Representation differences are structural, they are broader when concerning men. Contents differences focuses more on qualities, when women are concerned, and nursing care ends for men.

\section{INDEX}

Mots-clés : étudiant·e en soins infirmiers, représentations professionnelles, sexe, genre

Keywords : nursing students, professional representations, sex, gender

\section{AUTEURS}

\section{JEAN MAILLET-CONTOZ}

Doctorant en sciences de l'éducation, ED EPIClaboratoire ECP, Université Lyon 2

Thèmes de recherche : sexe et genre, représentations professionnelles, sociologie du genre. jmcontoz@yahoo.fr

\section{GILLES COMBAZ}

Professeur des universités, laboratoire ECP, Université Lyon 2

Thèmes de recherche : inégalités scolaires, éducation, sexe et genre, sociologie des rapports entre l'État et l'École, sociologie du curriculum, sociologie de l'encadrement intermédiaire au sein de la fonction publique.

gilles.combaz@univ-lyon2.fr 


\section{CHRISTINE MORIN-MESSABEL}

Professeure Laboratoire GRePS (EA 4163), Université Lumière Lyon 2

Thèmes de recherche : stéréotypes, sexe et genre, contexte éducatif, variables contextuelles et positionnelles, égalité des chances.

christine.morin-messabel@univ-lyon2.fr 